\title{
A Novel Method for Increasing the Overheat Ability of Radio Communication Devices with Frequency Hopping Spread Spectrum
}

\author{
https://doi.org/10.3991/ijim.v14i11.13647 \\ Amin Salih Mohammed \\ Lebanese French University, Erbil, Iraq \\ Salahaddin University, Erbil, Iraq \\ Igor Romanenko \\ Central Scientifically-Research Institute of Arming and Military Equipment \\ of the Armed Forces of Ukraine, Kiev, Ukraine \\ Saravana Balaji B ${ }^{(凶)}$ \\ Lebanese French University, Erbil, Iraq \\ saravanabalaji.b@gmail.com \\ Yevhen Kalashnikov \\ National University of Defense of Ukraine named after Ivan Chernyakhovsky, \\ Kyiv, Ukraine \\ Nina Kuchuk \\ National Technical University "Kharkiv Polytechnic Institute”, Kharkiv, Ukraine
}

\begin{abstract}
Due to increase in the number of radio devices with different types of signals and to increase the speed of information transmission, it is necessary to take into account the increase in the probability of erroneous reception of signals as a result of specific disturbances. The investigated methods (techniques) for increasing the noise immunity of radio communication with the FHSS do not take into account the influence of transmitters and fail to predict the load of the radio frequency spectrum. The purpose of this work is the development of the algorithm for the practical implementation of the method of increasing the noise immunity of the radio communication equipment with pseudo randomization of the operating frequency to increase the noise immunity of the radio communication equipment. The essence of the proposed algorithm, is the rational distribution of working frequencies between the radio communication devices with pseudo randomization of the operating frequency taking into account the mutual influence of transceivers on each other. According to the results of the research, the application of the methodology based on the proposed algorithm allows to increase the noise immunity of radio communication with pseudorandom interrupt of the operating frequency by an average of up to $30 \%$, depending on the channel state, while an increase in the computational complexity at the level of $10 \%$ is noted due to the increase in the number of computational procedures in the method. Thus, radio communication systems with the FHSS while working on
\end{abstract}


the basis of the proposed methodology shows an advantage over the known methods.

Keywords - Pseudorandom reconstruction, radio communication device, overheat ability, frequency hopping, spread spectrum

\author{
Abbreviations \\ FHSS is the frequency-hopping spread spectrum \\ $\mathrm{RCD}$ is the radio communication devices \\ $\mathrm{RCS}$ is the radio communication system \\ SCC is the signal code construction \\ RES is the radio electronic suppression \\ REW is the radio electronic warfare \\ TRN is the transmitter \\ $\mathrm{RCV}$ is the receiver \\ EMC is the electromagnetic compatibility \\ IRU is international radiocommunication union \\ IF is a intermediate frequency

\section{Nomenclature} \\ $\beta_{E}$ is the energy efficiency \\ $H(f)$ is the value of the signal entropy
}

$P_{1}$ is the average probability of error on bit at the effect of retransmitted interference

$P_{2}$ is the average probability of error on bit in the absence of retransmitted interferences at the input of the demodulator

$\Delta t_{\mathrm{ot}}$ is the operating time of the radio device at one frequency is less

$\Delta t_{\text {to }}$ is the total time of operation of the interference station

$\Delta t_{\mathrm{n}}$ is the delay time of interference

$G_{\Sigma}$ is the total spectral density of white Gaussian noise and intentional noise

$\rho$ is the coefficient characterizing the part of the frequency element damaged by the noise (overlap factor), $0 \leq \rho \leq 1$

$\gamma$ is the overlap factor

$r_{1}$ is the distance from the station of retransmitted interference to the RCD transmitter $r_{2}$ is the distance from the station of retransmitted interference to the RCD receiver $r_{12}$ is the distance between the transmitter and RCD receiver;

$C_{\mathrm{p}}$ is a speed of radio waves propagation

$P_{\mathrm{s}}$ is a signal strength

$M$ is a dimension of ensemble of signals

$R$ is the speed of the correction code $(R=k / n)$

$K$ is the number of information bits in the $n$ code combination length

$D$ is a value of code distance

$K_{\text {exp }}$ is a coefficient of expansion of the signal spectrum

$\Delta F$ is the bandwidth of the channel

$s_{\text {corr }}=(d-1) / 2$ is the error rate that the code corrects 
$J$ is the multiplicity of error in a block of $n$ elements

$C_{n}^{j}=n ! j !(n-j) !$ is the binomial coefficient equal to the number of different combinations of $j$ errors in the block of $n$ symbols

$X_{n}(f)$ is the normalized energy spectrum of the sample of the signal

$r_{s s}(n)$ is the correlation function.

\section{Introduction}

The problem of providing reliable communication in the conditions of the influence of directed and unintentional interference, as well as provision of multi-station access at work in packet radio networks can best be solved with the use of broadband signals in radio communication devices (RCD) [1]. The essence of the method of expanding the spectrum of signals is as follows: The expansion of the signal spectrum is a mode of transmission in which the signal occupies a band which is wider than the band that is the minimum necessary for the information transmission; the extension of the signal bandwidth is provided by a special code that does not depend on the transmitted information. For the next compression of the signal bandwidth and data recovery in the receiver, the RCD also uses a special code similar to the code in the transmitter and synchronized with it. One of the types of spectrum expansion used in the modern RCD is a frequency-hopping spread spectrum (FHSS) method [1-4].

For the FHSS method, the principle of combating noise is to place an information signal with a small dimension in a high-dimensional signal space. In such conditions, the noise generator should either distribute the limited power of interference over the entire frequency range, thereby creating a small spectral density of interference power, or use the entire power of the interference transmitter in a limited frequency range, leaving a part of the RCD frequency band free of interference. The typical shortcomings of the FHSS method are: low speed of data transmission and the absence of mechanisms for the fading warfare, resulting in error packets in the communication channel. Thus, the object of research is the process of formation of the FHSS signaling devices of radio communication in the context of deliberate interference. The subject of the research is the method of FHSS taking into account the interaction of transceivers, the strategy of the complex electronic warfare and prediction of the signal-interference situation. The purpose of the research is to develop a method of FHSS radio communication in the context of deliberate interference. To achieve this goal, the following tasks were solved:

- To develop the algorithm of the method of increasing the noise immunity of the RCD with the FHSS

- To evaluate the effectiveness of the proposed methodology in comparison with the known

- To elaborate practical recommendations on the use of the proposed methodology 


\subsection{Problem statement}

In science, the existing methods (techniques) for increasing the noise immunity of radio communication with the FHSS do not take into account the influence of the transmitters of RCD on each other, are not able to predict the frequency of radio frequency spectrum and are not able to change the law of adjustment of the operating frequency during the conduct of the radio communication, which significantly reduces the noise immunity of the RCD. In practice, a significant amount of destabilizing factors must be taken into account in order to increase the noise immunity of the RCD.

Objectives setting: Given parameters of the transmitter and the communication channel $\Psi=\left\{\psi_{\mathrm{i}}\right\}, i=\overline{1,9}$, where $\psi_{1} \ldots \psi_{9}$ is the speed of the FHSS $\mathrm{v}_{\mathrm{ch}}$, the maximum power of the useful signal $P_{s \max }$, the expansion factor of the spectrum of the $K_{\mathrm{s}}$ signal, the $\mathrm{v}_{\mathrm{i}}$ information transmission rate, the bandwidth of the $\Delta \mathrm{F}$ channel communication, a set of corrective codes with corresponding parameters.

It is necessary: To determine the values of the signal parameters (the speed of the FHSS, the parameters of the SCC, the coefficient of amplification of the spectrum of the signal, the transmitter power), at which the $\beta_{E}$ energy efficiency is maximized, while fulfilling the limits on the probability of false reception in the $P_{\text {error }} \geq P_{\text {error prob }}$. channel.

\section{Limitation:}

- The speed of the correction code $R=0,5-0,9$

- The dimension of the signal's ensemble $2 \leq M \leq 16$

- Coefficient of expansion $128 \leq K_{\exp } \leq 1024$

- Maximum probability of the signals error reception $P_{\text {error } \text { prob }}=10^{-5}$

- FHSS speed $100 \leq v_{\mathrm{ch}} \leq 10000$

Type of noise is noise frequency-manipulative interference in the part of the band, noise intermittent blocking interference, retransmitted interference (interference in response), which is an actual copy of the useful signal with time displacement.

\section{$2 \quad$ Related Work}

The most significant and fundamental work devoted to the disclosure of the properties of the FHSS method is the monograph [1]. It discusses in detail the advantages and disadvantages of this method, the basic mathematical models and analyzes the impedance of this method. However, no specific mechanisms for increasing the noise immunity of this method are proposed. Research is based on the use of frequencies in the FHSS signals, without considering other types of signal-code designs. In the article [2] an adaptive algorithm for the selection of operating frequencies for the RCD with FHSS in the conditions of intentional and unintended interference is considered. This algorithm is implemented on the basis of the theory-game approach. The disadvantage of this algorithm is that it is designed to work in the radio direction and is not suitable for the radio network operation. In the article [3], the method of automatic determination 
of the time parameters of radio signals from the FHSS on the background of narrowband noise is presented. The method is based on the analysis of the two-dimensional energy distribution of the signal mixture obtained using the window-modified modified Welch periodogram. The method allows to detect and remove third party narrowband radiation from the calculations, and to apply an algorithm for detecting abnormal values is filtering the false-defined duration of the frequency elements. The indicated method is not suitable for work with other types of interference and in the radio network of the RCD with the FHSS.

In the article [4] a method of dynamic selection of workers for industrial RCD with a FHSS is proposed. The essence of the proposed method is to evaluate the available frequency range by several standard indicators (probability of bit error and signal/noise ratio) and the selection of the best frequency subchannels for operation. The method is not intended to work in the conditions of intentional interference and does not allow for the mutual influence of transceivers on each other. In the article [5] a technique for the formation of signals from the FHSS in the conditions of the effect of intentional noise disturbances is proposed. The essence of the technique is to select the desired bandwidth of the signal FHSS (hopset), which provides the desired quality of information transmission. The author of this work limited himself to considering only one type of deliberate disturbance and does not take into account the mutual influence of the transmitters of the RCD on each other. In the article [6], the development of a signal formation method in radioactive drugs with FHSS in the transmission of speech in the conditions of the interference influence in response. The essence of the methodology is the location of the characters of speech frames in the interval of the frequency elements of the signal with the FHSS, in which the interference in response to the least important characters for speech reproduction, regardless of the type of signals transmitted.

In the article [7], an advanced method for selecting rational values of the parameters of multi-antenna systems of military radio communication with FHSS, depending on the signal-interference situation, was proposed. The mentioned technique allows to predict the signal-interference situation, to choose the most suitable working frequencies, and also allows not only to change the speed of the adjustment of the operating frequency, but also to change the appearance and duration of the expansion sequence, as well as the initial filling of the forming polynomial. The disadvantage of this methodology is that it does not allow to determine the mutual influence of the transceivers on the one hand, and is suitable only for radio direction. In the article [8], an algorithm for determining the time delay of signals from the FHSS was developed. The essence of the algorithm is to split the FHSS signal into separate frequency channels that correspond to the corresponding carrier frequencies. After that, the calculation of the mutual function of uncertainty in each channel occurs. The specified algorithm allows to increase the noise immunity of the RCD with the FHSS, but it cannot be used for work in the radio network.

However, the scientific results presented in known publications [1-8] have a number of disadvantages, namely: 
1. The choice of operating frequencies for the RCD with the FHSS occurs without taking into account the strategy of the radio-electronic suppression (RES) complexes and without taking into account the influence of the transmitters of the RCD on each other

2. Distribution of frequencies between radio communication devices with RCD is carried out on radio directions without taking into account the frequencies used in the radio communication system

3. Planning the use of frequencies between the RCD in the system occurs without taking into account the results of prediction of the effect of intentional interference on individual sections of the frequency range

4. The control of the parameters of the FHSS is carried out only by changing the speed of the adjustment of the operating frequency (without changing the law of the adjustment of the operating frequency)

5. Considered the impact of only one type of interference

6. A limited list of signal-code structures is used.

\section{Materials and Method}

An evaluation of the effectiveness and simulation of the proposed method of increasing the noise immunity of radio communication with the FHSS was proposed in the MatCad 14 software. In order to verify the adequacy and reliability of the developed method of increasing the noise immunity of RCD with FHSS, the developed method was performed by comparison with the results presented by the Iterative Solutions group [9]. Presented on this electronic resource of development allow to simulate characteristics of noise immunity of almost all known mobile data transmission systems. It should be considered that for the RCS one of the most unfavorable obstacles is the retransmitted interference (interference in response) [1, 6, 7]. The range of RES devices depends on many factors, including the power of radio transmitting devices of radio communication and repos equipment, the characteristics of their antenna systems, the sensitivity of receiving devices, the conditions of propagation of electromagnetic waves, types of radiation and methods of signal processing, the length of the working wave, methods of protection from obstacles. In addition, the range of means of the RES is influenced by the intensity of interference from local objects, the terrestrial (water) surface and non-earthly sources, the nature of the radiation and scattering of electromagnetic waves by the observed targets RCD. Considering all these factors is extremely difficult. In the regard, the range of RCD resonance impedance and the required power of the RES are evaluated mathematically by averaged parameters and specified in the simulation process. We should evaluate the station's interruptions during the broadcasting of disturbances in order to suppress the radio communication system. The termination of the retransmitted interference must coincide with the time at which the radiation of radio equipment signals ceases. The time required to perform the listed functions (the time when the relay interrupted station works) $\Delta t_{\text {to }}$ must be small enough so that the retransmitted interference has time to affect the radio receiver until the RCS transmitter is rebuilt to a different frequency. The result of the effect of retransmitted 
interferences on the receiver of the RCD in the general case can be estimated by the magnitude of the average probability of error on the bit of the $P_{\text {error }}$ information having the form [7]:

$$
P_{\text {error }}=\rho P_{1}\left(\frac{E_{s}}{G_{\Sigma}}\right)+(1-\rho) P_{2}\left(\frac{E_{S}}{G_{\Sigma}}\right)
$$

If the operating time of the RCD at one frequency $\Delta t_{\mathrm{ot}}$ is less than the total operating time $\Delta t_{\text {to }}$ and the delay time of the interference $\Delta t_{\mathrm{n}}$ due to the placement (topology) in the terrain of the transmitter and receiver of the RCS and station of retransmitted interference and the end speed of the radio wave propagation, then the retransmitted interference is ineffective.

The overlapping factor of the signal transmitted interference depends not only on the time of operation, but also on the relative location (topology) of the transmitter (TRN) and the receiver (RCV) of the RCS, as well as station relay interference on the ground, which determines the delay time delay $\Delta t_{\mathrm{n}}$ (fig. 1). If the radio device's operating time at $\Delta t_{\mathrm{ot}}$ one frequency is less than the $\Delta t_{\mathrm{tto}}$ total response time of the interference station and the delay time of interference $\Delta t_{\mathrm{n}}$, then the retransmitted interference is ineffective. In this case, the $\Delta t_{\text {ot }}$ permissible operating time of the receiver without affecting the noise is equal to it

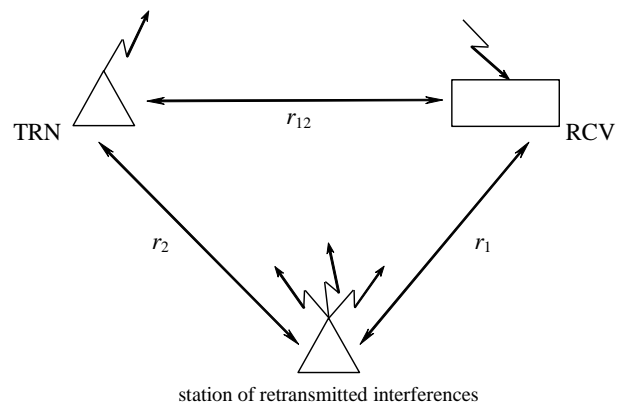

Fig. 1. Relevant location of the retransmitted interference station and RCS elements

$$
\Delta t_{o t} \leq \Delta t_{t t o}+\Delta t_{n} ; \quad \Delta t_{n}=\frac{1}{C_{p}}\left(r_{1}+r_{2}-r_{12}\right) .
$$

The closer the RCS receiver is to the station of retransmitted interference, the less allowable time for its operation $\Delta t_{\mathrm{ot}}$ without influence of interference. Effective effect of retransmitted interference at its sufficient power, on the RCS receiver is achieved when the ratio.

$$
(1-\rho) \Delta t_{o t} \geq \frac{r_{1}+r_{2}-r_{12}}{C_{p}}+\Delta t_{t t o}
$$


Using expression (3), it is possible to estimate the temporal possibilities of station of retransmitted interruptions at suppression of RCS with different duration of work at one frequency. In the article [6] the dependencies of the permissible working time $\Delta t_{\mathrm{ot}}$ of the RCS receiver without the influence of retransmitted disturbances on the $r=r_{1}+r_{2}-r_{12}$ distance for different values of the $\rho$ overlap factor is given. With the right choice of speed reorder frequency RCS $v_{\mathrm{ch}}=1 / \Delta t_{\mathrm{ot}}$ is the opportunity to completely get rid of retransmitted interference. Therefore, it is necessary to improve the existing scientific and methodical apparatus for controlling the parameters of systems and devices of radio communication with FHSS. The task of determining the parameters of the signal with the FHSS with the maximum energy efficiency indicators are reduced to a typical optimization problem. The system of equations for solving the optimization problem has the form

$$
\left\{\begin{array}{l}
\beta_{E}=F_{1}\left(v_{\mathrm{i}}, \Delta F, M, v_{c h}, R, d, P_{s}, K_{\text {exp }}\right) \rightarrow \max \\
P_{\text {error }}=F_{2}\left(P_{s}, M, R, d, K_{\text {exp }}, \gamma, \rho\right) \leq P_{\text {error prob }}
\end{array}\right.
$$

The system of equations for solving the optimization problem can be transformed into a form in

$$
\left\{\begin{array}{l}
\beta_{E}=\frac{v_{i}}{P_{s} / G_{n}}=\max , \\
100 \leq v_{c h} \leq 10000 \\
128 \leq K_{\text {exp }} \leq 1024 ; \\
P_{\text {error }}=\sum_{j=s_{\text {correct }}+1}^{n} C_{n}^{j} P_{\text {error }}^{j}\left(1-P_{\text {error }}\right)^{n-j} \leq \\
\leq P_{\text {error prob }} ; 2 \leq M \leq 16 .
\end{array}\right.
$$

The method of choosing the values of the parameters of the RCS signals with the FHSS, the algorithm of which is presented in fig. 2, consists of the following steps.

1. Entering the output data.

The parameters of the transmitter and the $\Psi=\left\{\psi_{\mathrm{i}}\right\}, i=\overline{1, m}$ communication channel and also the given speed of $v_{\text {prob }}$ information transmission are entered.

2. Distribution of frequency resource between the devices of radio communication

Recently, the urgent task of ensuring the quality functioning of radio equipment in common and adjacent bands of radio frequencies, which requires the development of new approaches to the analysis of electromagnetic compatibility radio equipment [10-11]. General approaches and separate methods of solving problems of this class are set out in the set of recommendations of the international radiocommunication union (IRU) [13]. But the proposed recommendations do not provide a 
comprehensive solution to the problem of the electromagnetic compatibility (EMC) analysis of special purpose radio communication.

The mobile radio service involves the use of a satellite, whose location locally changes, which complicates the EMC analysis [14]. The proposed procedure involves the use of a model of calculation containing a pair and group estimation with a simple logic of the mutual influence of the RCD in a group, which is based on a probabilistic (statistical) approach to evaluation [15]. A simple logic suggests that each of the RCD in a group can be considered as functionally independent of other devices. The calculation of EMC of the RCD is performed in the following order:

1. Selection of the RCD on a territorial basis, which are located within the limited research area.

2. Selection of the RCD selected on a territorial basis that potentially may interfere with the new RCD frequency appropriation on a frequency basis:

i. Identification of possible sources of noise over the main channel

ii. Identification of possible sources of noise along the first adjacent channel

iii. Determination of possible sources of intermodulation noise of the 3-rd order

iv. Calculation of the level of noise at the receiver input:

- On the main channel

- On the first adjacent channel

- Intermodulation of the 3-rd order.

3. Analysis of the results obtained and decision making based on the results of calculations on the possibility of frequency assignment, taking into account the effects of multiple noise or the need to choose another frequency.

To optimize the calculations, the analysis of EMC is proposed in two stages: preliminary and detailed. In the previous step, pre-selection and analysis of EMC for the specified frequencies are carried out.

In the EMC detailed analysis calculation is carried out taking into account the influence of the side radiation, the possibilities of blocking the receiver and the effect of intermodulation constituents.

The general algorithm for the assignment of operating frequencies and the EMC analysis for the RCD (figure 3) contains:

1. Entering the initial data.

2. Choosing a free fnew frequency within the calculated zone.

3. Selection of existing RCD within the calculated zone.

4. Selection of interference with hazardous RCD by frequency criterion.

5. Calculation of the level of $\mathrm{Pn}$ interference at the input of the receiver in the main and adjacent channels.

6. Analysis of the previous calculation results.

7. Calculation of the level of interference from the transmitter.

8. Analysis of the results obtained in 7-th part. 
9. Selection of RCD is carried out according to the criterion of proximity. For further calculations, RCD are selected within the radius of the service area.

10. 10. The calculation of possible interferences from the selected in 9-th part stations by adjacent and mirror channels, interferences on harmonics and intermodulation interruptions is carried out. The calculation of $\operatorname{Pn} \sum$ total noise for each station within the defined zone is carried out in accordance with the methodology given in [15] and contains the following stages:

- Calculation (measurement) of quantities and forms of spectra of noise at the receiver input.

- Calculation of the total capacity of interference at the input of the radio frequency amplifier.

- The definition of interconnection interferences that are formed at the output of the radio frequency amplifier products of intermodulation at frequencies of the main and mirror channels.

- Determination of the quantities and forms of the spectra products of intermodulation at the output of the radio frequency amplifier.

- Determination of the total power of intermodulation products at the output of the IF filter.

- Comparison of the calculated values of the total capacity of interference with allowable.

If the new frequency assignment leads to an overrun of the threshold by total interference, the transition to the selection of a new frequency (part 2) is carried out. A separate analysis of the object electromagnetic compatibility for the planned receiver of the RCD is carried out, analogously to 10 -th part. The required ratio is determined for the intended receiver, depending on the frequency selectivity of the receiver. If the total noise of a certain threshold is exceeded for interference, a transition to a new frequency is made (part 2). A positive conclusion about the possibility of frequency appointment is made.

Forecasting of signal-interference situation. The procedure described differs from those known in that it further comprises operations:

- Recirculation of incoming data for the one count

- Resampling the output process at logarithmic time scale

- Finding the energy spectrum of the received signal, determining the response

- Finding the entropy of the energy spectrum of the corresponding sample, which is subject to the resampling

- Calculation of the maximum value of entropy reviews

- Finding the forecast for implementing the maximum entropy value

- Resampling the result of the forecasting on the exponential time scale. 


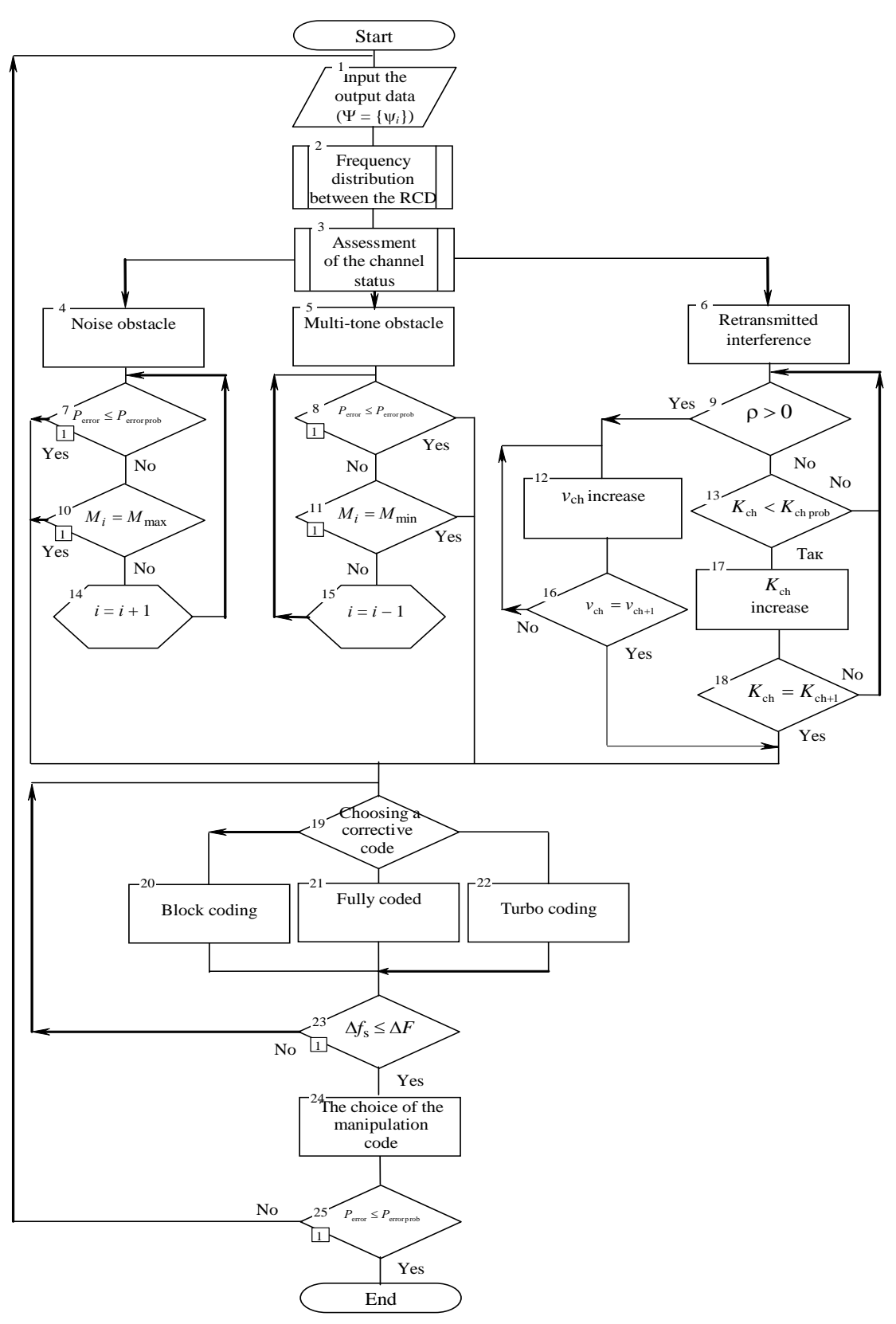

Fig. 2. Scheme of the algorithm for implementation of the method of controlling the parameters of RCS signals under the influence of intentional obstacles

The procedure for forecasting the signal-interference situation in the frequency range has the following sequence of actions [7]: 
1. There is an input of initial data.

2. Time compression of the process, which is predicted, is necessary for real-time signal processing. At the same time, at each step, the implementation is updated on a single countdown. Thus, a class of implementations, which differ from each other by one count, is formed. For forming a class of discrete counters, each implementation is subjected to a logarithm and sampling operation.

Then the maximum entropy value is found in accordance with the system:

$$
\left\{\begin{array}{l}
H(f)=-\int_{-1 / 2}^{1 / 2} \ln \left(X(f) / \int_{-1 / 2}^{1 / 2}(X(f)) d f\right) d f \\
X(f)=\sum_{n=-\infty}^{\infty} r_{s s}(n) \exp (-2 \pi f n) \\
X_{n}(f)=X(f) / \int_{-1 / 2}^{1 / 2}(X(f)) d f
\end{array}\right.
$$

Using the proposed procedure allows you to get a more accurate forecast than using another known procedure.

The choice of operating frequencies for radio communications in the conditions of deliberate interference is the choice of operating frequencies with a minimum probability of a bit error. This algorithm is based on the representation of the process of radioelectron confrontation in the form of two antagonistic systems. At the same time, decision-making on management is taken taking into account the analysis of the electronic environment and the criterion for minimizing the probability of a bit error. In order to implement this algorithm, the use of the coefficient of the working frequencies use by each antagonistic system (frequency overlapping frequency sub-channel blocking factor) was chosen.

The main stages of implementing the algorithm:

1. Determination of the type and characteristics of intentional obstacles.

2. Check the value of the overlap factor.

3. Formation of the game matrix of the radioelectronic conflict.

4. Formalization of the problem of linear programming.

5. Check the optimal control of the choice of operating frequencies.

6. Assessment of the state of the communication channel. At this stage, with the help of known methods [15-18], the spatial parameters of radiofrequency sources with the FHSS are estimated. In addition, the type and characteristics of intentional interference affecting the receiver input (noise, multi-tone interference or interference) are determined for a particular radio device.

7. Select the signal parameters. Depending on the type of interference acting on the receiver input, the positioning of the ensemble of signals, the frequency 
repositioning rate, and the magnitude of the expansion factor of the signal spectrum are selected.

In the known articles [18-20] in the conditions of deliberate interference, signal-code designs were selected by maximizing energy efficiency with restrictions on the value of the noise immunity for mean values of the signal-to-noise ratio in a discrete symmetric channel and the use of analytical dependencies for calculating the throughput of a discrete symmetric channel, effective only for the average signal-to-noise ratio corresponding to the probability of a $P_{\text {error }}<10^{-6}$ bit decoding error. In these articles, it is not taken into account that the devices of radio communication with FHSS in the conditions of intentional interference can operate at low signal-to-noise ratios in the channel and the probability of a $10^{-1} \ldots 10^{-4}$ bit decoding error. Considering that turbo code decoding algorithms are designed for "soft" input, it is necessary to use a "soft" demodulator in the RCD and consider discrete-continuous communication channels [21]. In order to calculate the probability of a $P_{\text {error }}$ bit error, it is recommended to use approximate formulas for large $Q_{0}^{2}$ signal/noise ratio (SNR) in the channel [21]. To analyze the characteristics of turbulence resistance of the turbo code used in channels with increased noise level, as well as in the presence of intentional interference in the communication channel, these formulas become inadequate in calculating. Therefore, we will use the exact analytical dependences to calculate the average probability of a bit error in the channel of communication with fluctuation noise for digital signal modulation methods that take into account the effect of intentional noise and, accordingly, would be effective for the calculation with small SNR in the channel.

Mathematical correlations for calculating the probabilities of a bit error for different types of signals and intentional interferences used to suppress the RCD with the FHSS are given in [22]. As shown in [22], in the vast majority of cases it is possible to identify several possible scenarios for the development of a disturbing situation. These scenarios can be matched with $\mathrm{N}$ different signal-code designs, which are appropriate to choose based on the parameters of the efficiency of the RCD under the influence of various types of intentional interference. Thus, with the influence on the radio communication devices of noise barrier and noise in the part of the band with increasing multiplicity of manipulation, impedance of signal reception improves (blocks 6, 9, 13). Under the influence of multi-tone interference to reduce the probability of a bit error, the position of the ensemble of signals must be reduced (blocks 7, 10, 14).

In promising stations of retransmitted interference in the UHF band using the latest technical advances and high-speed microprocessor technology in the RES equipment, the minimum response time can be tens of microseconds and less [6, 7]. In these conditions, an important parameter of an FHSS radio devices (in terms of noise immunity) is the actual operating time at the same frequency, this parameter determines the speed of the $v_{c h}$ frequency reorder, characterizing the ability of the radio vehicle with a FHSS to "escape" from the effects of retransmitted interference. The overlapping factor of the signal transmitted interference depends not only on the $\Delta t_{t t o}$ time of operation, but also on the relative location (topology) of the transmitter and receiver of the $\mathrm{RCD}$, as well 
as relay station on the ground, which determines the delay time of the $\Delta t_{n}$ interruption. Transmitted interference is ineffective only if the condition that the radio frequency at the one $\Delta t_{o t}$ frequency should be less than the total $\Delta t_{t t o}$ response time and the $\Delta t_{n}$ delay time of the interference caused by the placement (topology) on the terrain of the transmitter and the receiver of the RCD and the station of retransmitted interference and the final rate of radio waves propagation shall be less.

In this case, the coefficient $\rho=0$, and the average probability of the error per bit is determined only by the receiver's own noise. Under the action of relayed noise, the $\gamma$ overlap rate is checked. Depending on the $\gamma$ value, there is a change in the speed of resetting the operating frequency (step 11, 15). In the absence of relayed interference, and the mode of operation of the FHSS is chosen, in particular, the $K_{c h}$ coefficient, which varies depending on the signal/noise ratio according to the step-by-step law with the predefined gradation (stage 12,16,17), is chosen for the control of the noise interference in the part frequency and the noise interference.

Select the correction code: By type of noise-proof (corrective) codes, all SCC can be divided into two major classes: on the basis of block codes and on the basis of continuous codes. In addition, a separate class is a SCC based on cascading codes that use both block and continuous codes. Recently, much attention has been paid to a new and promising class of noise immunity codes are parallel cascading coaxial codes known as turbo codes [21, 22]. The introduction of turbo codes opened a new direction in solving the problem of creating effective codes and decoding them with little complexity. These codes allow to provide noise immunity to the reception of signals by channels with noises close to the theoretically possible (Shennon's boundary). From [21-23] it is seen that the most effective at present correction codes are turbo and low-density codes. Each of them has its advantages, disadvantages and, accordingly, its field of application. For example, turbo and low-density codes are capable of operating at a power level of the channel, only a few tenths of a decibel higher than its throughput. The use of turbo codes during the construction of the SCC allowed for additional energy gain of the signal/noise ratio for channels with fluctuating noise and fading compared to circuits using circuits. The use of noise immunity encoding increases the width of the spectrum of the signal, so the code parameters are chosen depending on the bandwidth of the communication channel $\Delta \mathrm{F}$.

Selecting the manipulation code: While coordinating the codec of the binary noise immunity code and the modem of multi-position signals, it is necessary to use a manipulation code, in which a greater distance between Hamming's and the code combinations corresponds to a greater distance between the Euclidean signals corresponding to them. Methods for coordinating modulation and coding can be divided into two groups: matching with optimal manipulation code and matching on the basis of the partition of the ensemble into nested subassemblies. The SCCs belonging to the first group are the result of matching the known binary noise immunity codes with the multi-stationed ensemble of signals by using a specially selected manipulation code. Since errors often occur due to conversions in neighboring signals, then the code combinations that match the neighboring signals should be the smallest number of binary characters. This requirement in some cases satisfies the Gray code [22, 23]. 
The second group includes a fairly large number of types of SCCs that differ in modification of matching methods. The basis for constructing a SCC of this kind is the partition of an ensemble of signals into embedded subassemblies [22, 23]. The partition is carried out in such a way that the partial ports have the same number of signal points. Euclidean distances $\mathrm{dE}$ between the neighboring signals of the subassemblies are the same, and the minimum distances $\mathrm{dE}$ min between the signals of the subassembly are increased with each step of the partition. Checking compliance with requirements to ensure noise immunity to receive signals. After selecting the manipulation code, the requirements for ensuring the given probability of false signal reception are checked. If the $P_{\text {error }}$ requirements are not met, then there is a need to change the source data. In the event that a $P_{\text {error }} \leq P_{\text {error prob }}$ restriction is performed for several SCCs, the SCC with the maximum values of $\beta_{\mathrm{E}}$ is selected.

\section{$4 \quad$ Experiments}

In order to evaluate the effectiveness of the proposed methodology, simulation modeling of the RCD with FHSS was carried out at different degrees of deliberate interference affecting the communication channel. Simulation was conducted in the MathCad [24] software environment using the mathematical relationships obtained above. Fig. 3 shows a graph of dependence of the probability of a bit error on the signal/noise ratio (for the case of noise barrier interference with frequency manipulation $\rho=1$ ). Simulation was carried out in the following conditions:

Devices of radio communication with the FHSS: Frequency range is $30-512 \mathrm{MHz}$; transmitter power is $10 \mathrm{~W}$; the bandwidth of the emitted frequency is $12.5 \mathrm{kHz}$, the receiver sensitivity is $110 \mathrm{~dB}$; the number of RCD in the network-4; number of frequency channels for resetting is 10,000; the number of rebuilding is 333, 5 jumps / sec.

Radio-electronic suppressor complex: Frequency range is $30-2000 \mathrm{MHz}$; transmitter power is $2000 \mathrm{~W}$; maximum bandwidth that can be suppressed is $80 \mathrm{MHz}$; the number of radio lines with the FHSS, which can be suppressed simultaneously-4, type of noise is the noise barrier with frequency manipulation; strategy of the complex is REW-dynamic.

To verify the effectiveness of the proposed method, let us suppose that the time of operation of the RCD with the FHSS at the same frequency is the same with the REW complex. Briefly we will describe the graphic relationships, obtained in fig. 3 


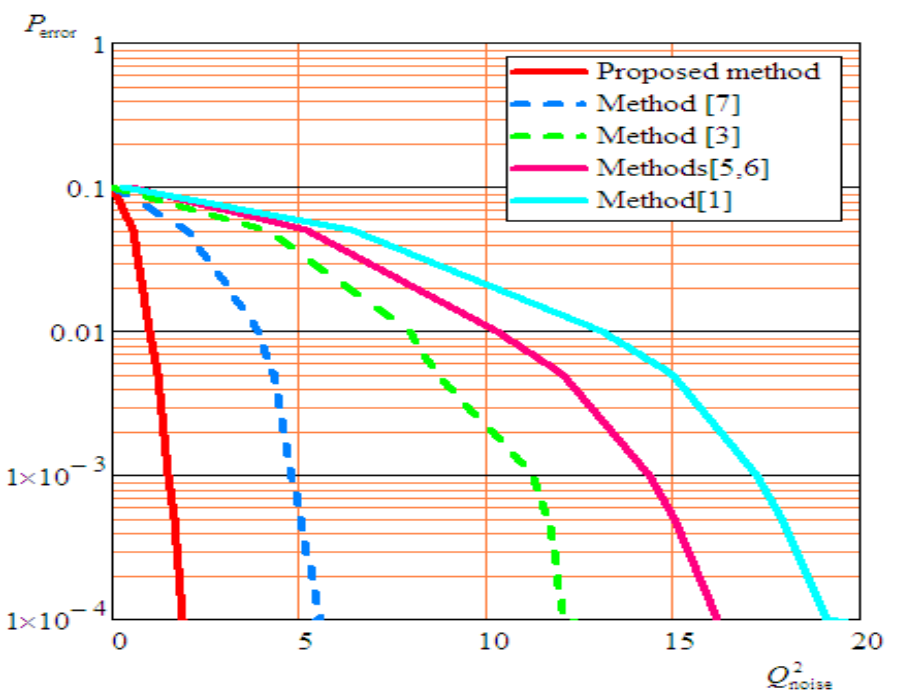

Fig. 3. Dependence of the probability of bit error on the signal-noise ratio for different methods under the influence of fluctuation noise and noise barrier interference $(\rho=1)$

As shown in fig. 3 , the best ratio of the error probability of the signal/noise ratio is proposed by the authors of the technique. The indicated graphic dependencies were obtained by averaging the signal/noise ratio on the frequency subchannels. This advantage is due to the prediction of the strategy of the REW complex taking into account the influence of transmitters on each other and the selection of optimal SCCs for a separate frequency subchannel. Compared with the method [1], the proposed technique has an average gain of $14.17 \mathrm{~dB}$.

Figure 4 shows a graph of the dependence of the bit error probability on the sig$\mathrm{nal} /$ noise ratio (for the noise noise in the band portion with frequency manipulation $\rho=0,5)$.

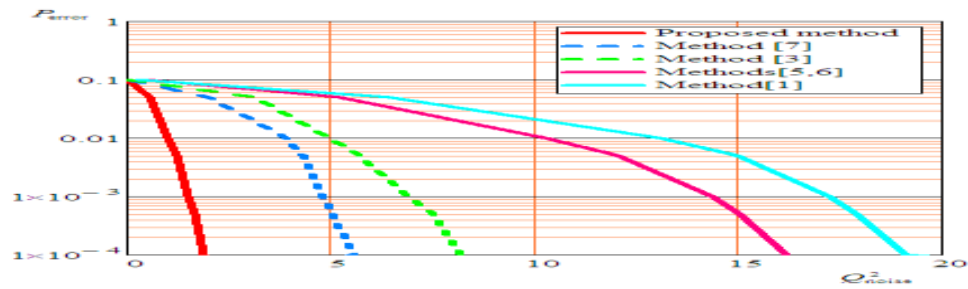

Fig. 4. Graph of probability dependence of the bit error on the signal/noise ratio (for the case of noise in the part of the band with frequency manipulation $\rho=0.5$ )

From the ones shown in the fig. 4 graphic dependencies it can be concluded that the method proposed by the authors of the article provides approximately the same impedance to the influence of various types of intentional noise. A practical test was conducted to test the effectiveness of the developed methodology, by the developing 
software Python 3.6 (Jupyter-notebook). The software was installed on a PC with 4 programmable LimeSDR transceivers connected to the GNU Radio software and connected to the noise generator RIGOL DG5252 that mimics the operation of the REW complex. Fig. 5 shows the work of 4 transceivers according to the methodology and noise generator RIGOL DG5252. As can be seen from fig.5 that the devices of the REW is not in a position to carry out the guaranteed suppression of radio communication with the FHSS using this method. For greater clarity fig. 6 shows an enlarged image of the work of the indicated method. From the fig. 6 it is possible to conclude that the devices of the REW narrowband interference affected about $10 \%$ of the hopset, which can be effectively corrected by corrective codes.

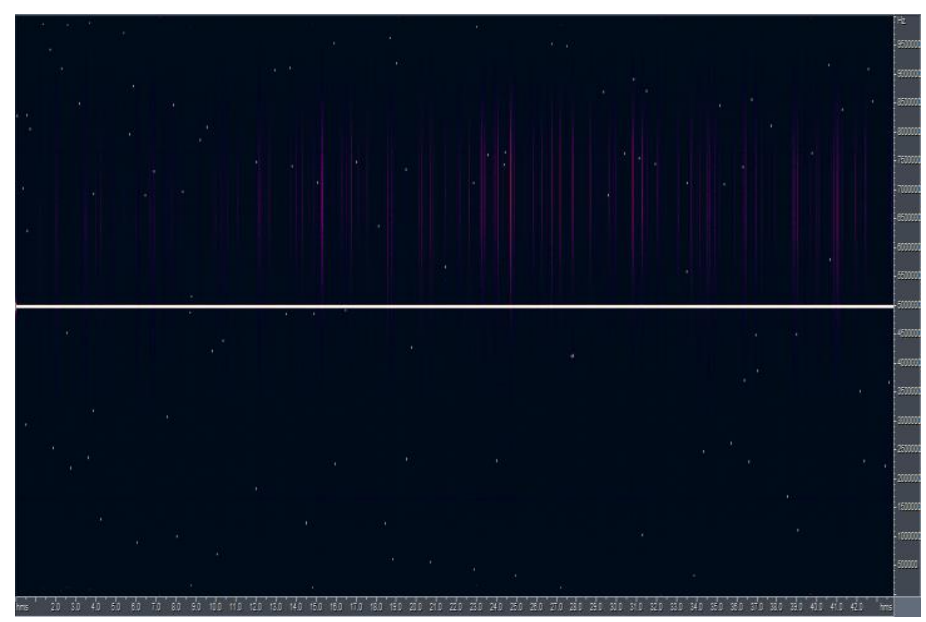

Fig. 5. Operation of 4 transceivers according to the specified procedure and noise generator RIGOL DG5252

Estimation of the computational complexity of the implementation of the developed method showed that for the given output data and while using the ADSP-21261 processor, the formation of a signal with optimal parameter values can be carried out in real time with the consideration of the delay required to transmit information about these values through the service feedback channel. The research of the developed algorithm of adaptive RCD functioning according to the developed method showed that the adaptive scheme of the radio communication devices provides on average 16-27\% higher energy efficiency (coefficient of power use of the signal) than nonadaptive. 


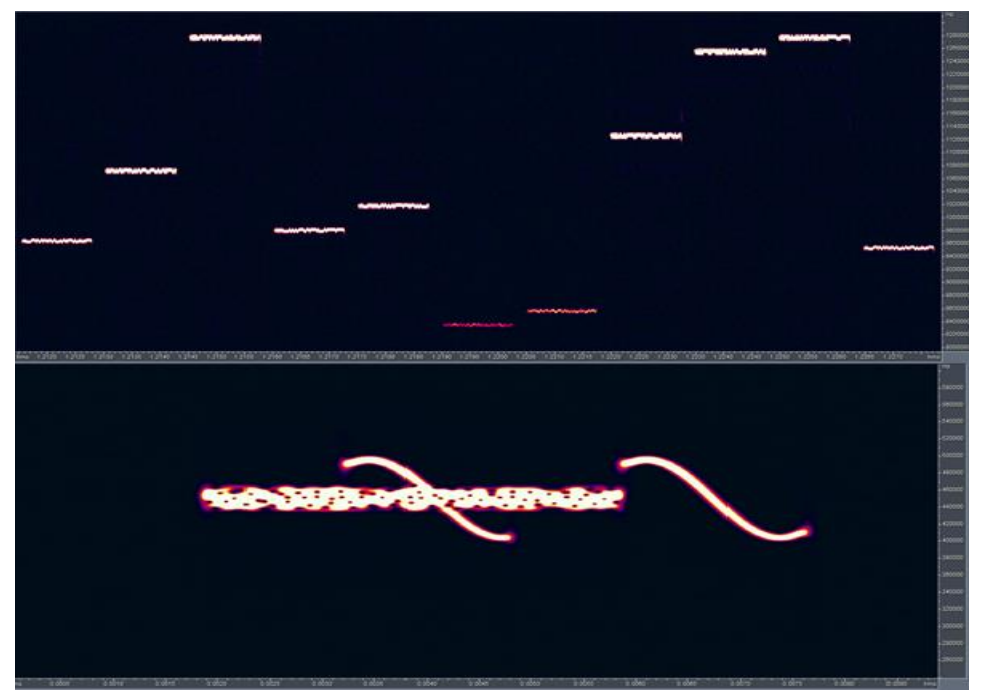

Fig. 6. Operation of 4 transceivers according to the specified methodology and noise generator RIGOL DG5252

Figure 7 shows the simulation of the work of the RCD and the devices of the REW under the influence of noise disturbance with frequency manipulation.

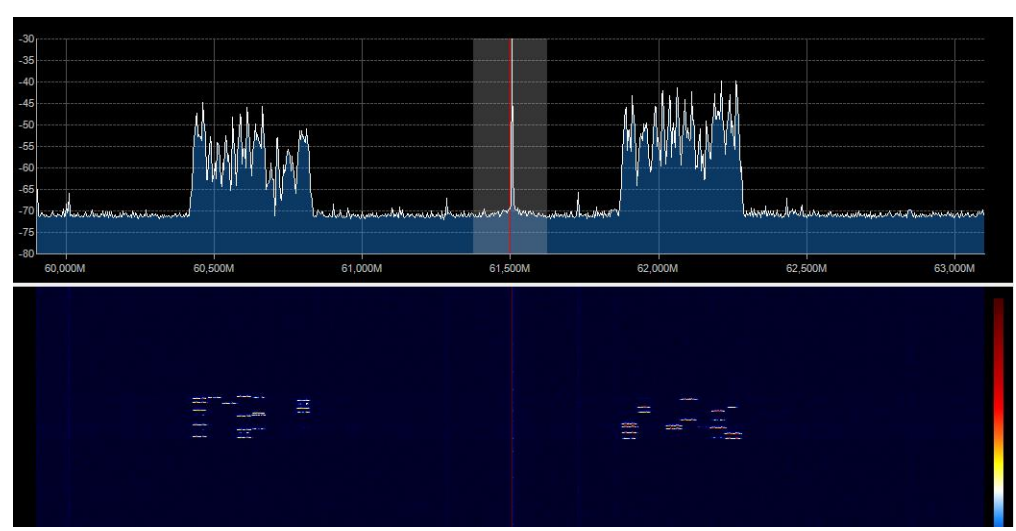

Fig. 7. Simulation of the RCD work and the devices of the REW

\section{Discussion}

The article proposes a method of increasing the noise immunity of radio communication equipment with pseudorandom reconstruction of the operating frequency. The difference between the proposed methodology, from the known, defining its novelty are: 
- The choice of operating frequencies for the RCD with the FHSS takes place taking into account the strategy of the radio-electronic suppression complexes and taking into account the influence of the transmitters of the RCD on each other

- The frequency distribution between the radio communication equipment and the FHSS is based on the number of the RCDs operating on the network

- Frequency planning is divided into two stages - preliminary and detailed. At the previous stage, the choice and analysis of electromagnetic compatibility of the RCD with the FHSS on the frequency-territorial basis is carried out. At the stage of detailed analysis, calculations of electromagnetic compatibility are carried out taking into account the possible influence of side and intermodulation radiation, as well as the possibility of blocking the receivers when the group is located in a limited space

- The control of the parameters of the FHSS is carried out by changing the speed of the adjustment of the operating frequency and, if necessary, changing the law of the adjustment of the operating frequency

- Taking into account the influence of the most common types of deliberate disturbances

- Using positional $M$ signal-code structures

The evaluation of the proposed method effectiveness showed an increase in noise immunity of radio communication equipment with a FHSS during its use. The proposed methodology is advisable to use in a developing software for modules (blocks) for evaluating perspective radio communication equipment based on the open interface architectures of version SCA 2.2, which will allow:

- To use effective signal-code designs to ensure the noise immunity of channels

- To ensure efficient use of the radio frequency resource of programmable radio communication equipment

- To increase the rate of communication channels estimation

- To reduce the use of computing resources of radio communication with programmable architecture.

\section{Conclusion}

In this work, practical implementation of algorithm for realization of the increasing the noise immunity method of radio communication devices with the pseudorandom reconstruction of working frequency is shown. The frequency distribution between the radio communication equipment and the FHSS is based on the number of RCDs operating on the network. Frequency planning is divided into two stages - preliminary and detailed. At the preliminary stage, the choice and analysis of electromagnetic compatibility of the RCD with the FHSS on the frequency-territorial basis is carried out. At the stage of detailed analysis, calculations of electromagnetic compatibility are carried out taking into account the possible influence of side and intermodulation radiation, as well as the possibility of blocking the receivers when the group is located in a limited space. The control of the parameters of the RCD is carried out by changing the speed of the adjustment of the operating frequency and, if necessary, changing the law of the 
adjustment of the operating frequency. The proposed method can be implemented in radio communication with programmable architecture. To do this, it is necessary to adapt the signal processor through additional software for a specific radio communication device. It is advisable to develop the software, for example, on the SCA 2.2 platform.

\section{$7 \quad$ References}

[1] Information technology (1995) Vocabulary. Part 28. Artificial intelligence. Basic concepts and expert systems: ISO/IEC 2382-28:1995, Geneve, $36 \mathrm{p.}$

[2] Automated systems (1993). Terms and designation: DSTU 2226-93, State standard of Ukraine, Kyiv, 86 p., available at: http://online.budstandart.com/ua/catalog/doc-page.html? id_doc $=61937$ (in Ukrainian)

[3] Subbotin S. O. and Olijnyk A. O., (2006), The method of selecting information features for product diagnostics, Ukraine patent, No. u200603087; bulletin 4 p. (in Ukrainian)

[4] Akaike H., (1974), A new look at the statistical model identification, IEEE Transactions on Automatic Control, Vol. 19, no. 6, pp. 716-723. https://doi.org/10.1109/tac.1974.1100705

[5] Babak O. V. and Tatarinov A. E., (2005), About one approach to solving classification problems in conditions of incomplete information, Cybernetics and Systems Analysis, No. 6, pp. 116 - 123. (in Ukrainian)

[6] Slyusar V. I., (2008), NATO Military Communications: Problems of Modern Technologies, Electronics: Science, Technology, Business, no. 4, pp. 66 - 71. (in Ukrainian)

[7] Slyusar V. I., (2010), Data transmission from the UAV: NATO standards, Electronics: Science, Technology, Business, no. 3, pp. 80 - 86. (in Ukrainian)

[8] Slyusar V. I. (2010), UAV radio links: implementation examples, Electronics: Science, Technology, Business, no. 5, pp. 56 - 60. (in Ukrainian)

[9] Romanyuk V.A., Stepanenko E.O., Panchenko I.V. and Voskolovich O.I., (2017), Self-organizing flight radio links, Scientific works of Telecommunications and Information Military Institute, no 1, pp. 104 - 114, available at: http://www.viti.edu.ua/files/zbk/2017/14 1 2017.pdf (in Ukrainian)

[10] Romanyuk V.A. and Stepanenko E.O., (2017), Synthesis problems of the networks topology of mobile components with using telecommunication aerolattforms Scientific works of Telecommunications and Information Military Institute, no 3, pp. 149 - 157, available at: http://www.viti.edu.ua/files/zbk/2017/3/17_3_2017.pdf (in Ukrainian)

[11] Suyi L., Wang S., (2006), Machine health monitoring and prognostication via vibration information, Intelligent systems design and applications, Sixth international conference, Jinan, 16-18 October 2006, IEEE, Los Alamitos, p. 879. https://doi.org/10.1109/isda.2006. $\underline{188}$

[12] Subbotin S. O., (2012), Diagnostic synthesis software resources for use cases, current problems and achievements in the telecommunications and information technology industry, VI International Scientific and Practical Conference Zaporizhzhia Polytechnic National University, Zaporizhzhja, pp. 21-22. (in Ukrainian)

[13] Neagu C.D., (2000), Using artificial neural networks in fuzzy reasoning, abstract of the dissertation, University of Galati, Galati, $42 \mathrm{p}$.

[14] Snytjuk V. Je., (2009), Evolutionary decision-making technologies in an uncertainty conditions, abstract of the thesis of Doctor of Technical Sciences, The Institute of Mathematical Machines and Systems Problems of the Ukraine National Academy of Science, Kyiv, 36 p. (in Ukrainian) 
[15] Li S., (1995), Automated tool condition monitoring in machining using fuzzy neural networks, PhD thesis, McMaster University, Hamilton, 187 p.

[16] Voronkin R. A., (2004), Mathematical modeling of genetic search processes to improve the learning quality of direct distribution neural networks, $\mathrm{PhD}$ thesis of Technical Sciences, Stavropol, 237 p. (in Ukrainian)

[17] Abraham A., Grosan C. and Pedrycz W., (2008), Engineering evolutionary intelligent systems, Springer, Berlin, $444 \mathrm{p}$.

[18] Boguslaev A. V., Olejnik Al. A., Olejnik An. A., Pavlenko D. V. and Subbotin S. A., (2009), Advanced modeling technology optimization and intelligent automation of aircraft engine life cycle stages, Motor Sich, Zaporizhzhja, 468 p. (in Ukrainian)

[19] Klyuev V. V., Sosnin F. R. and Filinov V. N., (1996), Engineering: Measurement, control, testing and diagnostics, encyclopedia, Vol. III-7, 464 p. (in Ukrainian)

[20] UCI machine learning repository, available at: http://archive.ics.uci.edu/ml/datasets/

[21] Shuvakin Yu. A., (2016), Simulation of the kinematics and flight dynamics of an unmanned aerial vehicle, Problems of modern science and education, Olympus, Ivanovo, No. 16 (58), pp. $44-47$.

[22] Lebedev A. A. and Chernobrovkin L. S., (2010), Dynamics of flight of unmanned aerial vehicles, High Schools, Moscow, $618 \mathrm{p}$.

[23] Chernodub A. N., (2012), Training of neuroemulators with use of pseudoregularization for model reference adaptive neurocontrol, Intelligent systems, no. 4, pp. $602-614$. https:// doi.org/10.1109/icumt.2012.6459698

[24] Mu C., Wang D. (2017), Neural-network-based adaptive guaranteed cost control of nonlinear dynamical systems with matched uncertainties, Neurocomputing, vol. 245, pp. $46-54$, DOI: https://doi.org/10.1016/j.neucom.2017.03.047

[25] Lin Z., Ma D., Meng J., Chen L., (2018), Relative ordering learning in spiking neural network for pattern recognition, Neurocomputing, vol. 275, pp. 94 - 106, DOI: https://doi. org/10.1016/j.neucom.2017.05.009

[26] Yu J., Sang J., Gao X., (2017), Machine learning and signal processing forbig multimedia analysis, Neurocomputing, vol. 257, pp. 1-4, DOI: https://doi.org/10.1016/j.neucom.2017. $\underline{01.091}$

[27] Lv Y., Na J., Yang Q., Wu X., Guo Y., (2016), Online adaptive optimal control for continuous-time nonlinear systems with completely unknown dynamics, International Journal of Control, vol. 89, pp. 99 - 112, DOI: https://doi.org/10.1080/00207179.2015.1060362

[28] Sun Y., Xue B., Zhang M., Yen G. G., (2018), Automatically Designing CNN Architectures Using Genetic Algorithm for Image Classification, Cornell University, available at: https://arxiv.org/abs/1808.03818

[29] Zela A., Klein. A., Falkner S., Hutter F., (2018), Towards Automated Deep Learning: Efficient Joint Neural Architecture and Hyperparameter Search, Cornell University, available at: https://arxiv.org/abs/1807.06906

\section{Authors}

Amin Salih Mohammed received the bachelor's and master's degrees from the Kharkiv National University of Radio Electronics, and the Ph.D. degree from the Kharkiv National University of Radio Electronics, in 2012. He is currently a vice-president for scientific affairs of Lebanese French University and Assistant Professor with the Department of Computer Networking, Lebanese French University, and the University of Salahaddin - Erbil. He has published over 40 articles in international and 
national journals and conferences. His fields of interests include wireless networks, ad-hoc networks, and information security. ORCID: https://orcid.org/0000-0002-29936182. Email: kakshar@1fu.edu.krd, amin.mohammed@su.edu.krd

Romanenko Igor, Doctor of Technical Sciences, Professor, Leading researcher of the scientific research department of Central scientifically-research institute of arming and military equipment of the Armed Forces of Ukraine, Kiev, Ukraine The Military Command Academy of the Air Defense Forces named after G.K. Zhukov in 1981 for command and staff operational work; the Academy of the Armed Forces of Ukraine in 1996 for the specialty "Integration and association of the Armed Forces". Research interests: unmanned aviation complexes; control and data transmission systems; noise immunity.ORCID: https://orcid.org/0000-0001-5339-7900, e-mail: igor_romanenk@ukr.net.

Saravana Balaji B working as Assistant Professor, Department of Information Technology, College of Engineering \& Computer Science, Lebanese French University, Erbil, Kurdistan, Iraq. He has completed undergraduate, postgraduate and doctorate engineering degree in Computer Science and Engineering. So far, he has published more than 30 research articles in various reputed international journals and conferences.

ORCID: https://orcid.org/0000-0001-9077-1658, email: saravanabalaji.b@gmail.com

Kalashnikov Yevhen, PhD, Chief of Research Laboratory, Research laboratory (problems of development of combat use of rocket forces and artillery), National University of Defense of Ukraine named after Ivan Chernyakhovsky. In 2006 he graduated from the National Defense University of Ukraine named after Ivan Cherniakhovsky, in 2014 he defended his dissertation for the Ph.D degree. Research interests: unmanned aviation complexes; control and data transmission systems; ORCID: https://orcid.org/0000-0003-4552-6439, e-mail: evg_kalas@ bigmir.net.

Kuchuk Nina in 2011 graduated from National Technical University "Kharkiv Polytechnic Institute" with a degree in Economic Cybernetics, in 2014, she defended her thesis and received a PhD. Since 2014, she has worked as an associate professor at V. N. Karazin Kharkiv National University. Since 2019 she has been working as an Associate Professor in the Department of Computer Engineering and Programming of National Technical University "Kharkiv Polytechnic Institute". Research interests is an Information Technology and radio communication. ORCID: https://orcid.org/00000002-0784-1465, e-mail: nina_kuchuk@ukr.net

Article submitted 2020-02-08. Resubmitted 2020-03-26. Final acceptance 2020-03-29. Final version published as submitted by the authors. 